\title{
Politique
}

\section{Changement et insertion des femmes dans le système politique}

\section{Carolle Simard}

Numéro 5, hiver 1984

Femmes et pouvoir

URI : https://id.erudit.org/iderudit/040444ar

DOI : https://doi.org/10.7202/040444ar

Aller au sommaire du numéro

Éditeur(s)

Société québécoise de science politique

ISSN

0711-608X (imprimé)

1918-6584 (numérique)

Découvrir la revue

Citer cet article

Simard, C. (1984). Changement et insertion des femmes dans le système politique. Politique, (5), 27-49. https://doi.org/10.7202/040444ar d'utilisation que vous pouvez consulter en ligne.

https://apropos.erudit.org/fr/usagers/politique-dutilisation/ 


\title{
Changement et insertion des femmes dans le système politique*
}

\author{
Carolle SIMARD \\ Université du Québec à Montréal
}

\begin{abstract}
Il semble exister de nombreuses explications de la faible insertion des femmes dans le système politique. Ces explications relèvent tantôt de facteurs sociaux et culturels, tantôt de facteurs institutionnels. Sur cette question, l'étude comparative de Maurice Duverger, quoique relativement ancienne sur les femmes et la vie politique, constitue de nos jours un important ouvrage de référence ${ }^{1}$. Au niveau canadien et québécois, des recherches plus récentes ont identifié certains des multiples obstacles à l'insertion des femmes dans le système politique ${ }^{2}$. Après l'histoire, la science politique a décrit, parfois analysé, comment tantôt le droit et les institutions,
\end{abstract}

* Je remercie André Bernard, Micheline de Sève et Jean-Jacques Gleizal pour leurs commentaires sur une première version de ce texte. 1955.

1. Maurice Duverger, La participation des femmes à la vie politique, Paris, Unesco,

2. Voir ces études:

Jerome H. Black et Nancy E. McGlen, «Male-Female Political Involvement Differentials in Canada, 1965-1974" Canadian Journal of Political Science, vol. 12, septembre 1979, 471-497.

Evelyne Tardy, La politique: un monde d'bommes?, Montréal, Hurtubise HMH, 1982. Jill Vickers et Janine Brodie, The Politics of the Second Electorates Women and Public Participation, eds. Joni Lovenduski and Jill Hills, London: Routledge and Keagan Paul, 1981.

Jill Vickers et Janine Brodie, Canadian Women in Politics: An Overview, Ottawa, Cahier de l'ICRAF, no 2, 1982. 
comment tantôt les faits, ont organisé l'exclusion des femmes des organes politico-administratifs qui dirigent l'État. Sans doute, nombre d'obstacles institutionnels et sociaux sont analogues à ceux qu'on identifie pour expliquer les causes de la faible insertion de la classe ouvrière dans le système représentatif. Mais les obstacles cités et les explications proposées ne sont ni structurés dans un ensemble cohérent, ni hiérarchisés; il demeure extrêmement difficile de mesurer l'importance respective et relative de chacun et de chacune. Bref, on ne sait toujours pas s'il existe des obstacles décisifs ou encore s'il existe une combinaison d'obstacles, combinaison qui suffit à exclure les femmes ou encore à limiter leur insertion. La question de l'exclusion/inclusion des femmes hors et dans le système politique en appelle une seconde, logiquement première: qu'y a-t-il de spécifique à l'exclusion des femmes? $\mathrm{Ou}$ encore est-ce l'institutionnalisation du politique qui a donné lieu à l'exclusion des femmes ou n'est-ce pas plutôt de l'exclusion des femmes qu'a découlé une structuration spécifique du champ du politique d'abord, du champ de la science politique ensuite?

Aborder de front cet ensemble de questions serait téméraire. D'autant que la quasi-totalité des études disponibles posent le problème, important mais limité, de la relative exclusion des femmes du système représentatif en général et du processus décisionnel en particulier. De ces travaux, il ressort notamment que l'augmentation de la participation électorale des femmes ne s'est nullement traduite par leur intégration au cercle gouvernemental, à tous les niveaux. Certes, les femmes participent plus qu'auparavant au sein des assemblées parlementaires ${ }^{3}$, mais leur participation qualitative va dans un sens particulier. Précisément, les femmes intégrées dans le cercle gouvernemental sont spécialisées dans un certain nombre de questions considérées par l'opinion courante

3. En plus des ouvrages pré-cités voir:

Pierre Drouilly, «Les femmes et les élections», Le Devoir, 17 décembre 1980. 
comme spécifiquement féminines. Cette tendance semble s'accentuer avec le temps, d'autant qu'on la retrouve partout: dans l'administration, dans les partis, dans les groupes et les mouvements, dans les syndicats, dans l'entreprise privée.

Mais, ces travaux montrent aussi le cercle vicieux politique dans lequel la majorité des femmes semblent enfermées. Pour la plupart, elles demeurent condamnées à l'absence et au conservatisme, en dépit des mesures législatives et réglementaires censées favoriser leur intégration à tous les niveaux et dans tous les lieux, notamment parce que ces mesures sont demeurées lettre morte dans l'ensemble.

La spécificité de l'exclusion/inclusion des femmes nous force donc à aller au-delà de l'analyse institutionnelle et à aborder cette question à plusieurs niveaux. En effet, les femmes participent à la vie politique. En un sens, elles ne sont pas totalement exclues du système représentatif. Leur exclusion/inclusion varie selon les secteurs d'activité politique. Dans les comités de parents, parmi les travailleurs bénévoles des partis comme des associations volontaires, c'est une majorité de femmes qui constituent l'infrastructure. Mais elles ne participent guère au processus final de la décision politique; elles demeurent exclues de l'exercice du pouvoir politique puisque les enjeux sociaux et économiques sont arrêtés en des lieux et à des niveaux dont elles sont absentes. N'est-ce pas là une des spécificités du processus d'exclusion/inclusion des femmes? Pour en rendre compte, nous traiterons de la a'destion à l'aide d'indices qui se situent à des plans différents. Puisque la relative intégration des femmes dans le système représentatif ne constitue qu'une facette du rapport spécifique des femmes au politique. Ce rapport est dual, contraire. D'un côté, le politique rejette les femmes, au mieux les intègre à des niveaux et dans des lieux circonscrits; mais d'un autre côté, le politique est bouleversé. La politique des femmes étend le champ du politique au «vécu» et suppose donc une remise en cause de la vie politique traditionnelle. 
Notre question matrice sera abordée et discutée à l'aide de trois interrogations principales. La première concerne la place des mécanismes électoraux et du système partisan dans le système représentatif. La seconde est celle de la place du système représentatif dans le système politico-administratif. La troisième analyse la place du système politico-administratif dans la science du politique. Certains jugeront cette dernière partie risquée, voire téméraire. Néanmoins, la faiblesse relative du schéma explicatif dominant quant à la question de l'insertion des femmes dans le système politique nous a conduit à lire ce problème à travers l'ébauche d'un autre modèle d'interprétation.

Sans aucun doute, la question de la spécificité de l'exclusion des femmes ne sera pas tranchée pour autant. Mais peut-être serat-elle moins trouble, même si dans l'ombre continueront de subsister une foule d'interrogations. N'en doutons pas, ces interrogations susciteront de nouveaux énoncés, d'autres hypothèses.

\section{Mécanismes électoraux et systèmes partisans/système représentatif}

La nature et la forme de la relative intégration des femmes dans le système représentatif semblent partiellement déterminées par les mécanismes électoraux. Voilà une des explications que donne la science politique à propos de la faible intégration des femmes dans le système politique. Celui-ci est structuré à travers des mécanismes électoraux qui fixent les conditions de la participation aux élections ainsi que les modalités de cette participation. Ces mécanismes opèrent dans le cadre d'un système de partis, système qui, selon Duverger, constitue «un élément important des institutions politiques d'un pays " ${ }^{4}$.

Pour aborder la question de l'exclusion/inclusion des femmes hors et dans le champ politique, il convient donc de considérer

4. Maurice Duverger, Institutions politiques et droit constitutionnel, Paris, PUF, 1970,151 . 
les institutions politiques puisque la vie politique est d'abord la vie parlementaire et représentative.

Les données disponibles sur la présence des femmes dans les institutions parlementaires nous apprennent que les femmes y sont peu nombreuses; que les femmes accèdent aux institutions parlementaires dans une proportion moins faible qu'auparavant; qu'elles atteignent certains niveaux de représentation mais demeurent limitées à des secteurs de la vie parlementaires. Si nous passons sur le détail de ces données, ce n'est nullement parce que nous attribuons peu d'importance aux chiffres. Sans doute, la faiblesse relative des femmes dans la vie parlementaire déterminet-elle, du moins partiellement, le caractère incomplet de leur intégration à la vie politique. Mais nous nous intéressons davantage aux mécanismes censés expliquer cette faiblesse. Parce que, nous dit-on, ils constituent des obstacles à l'intégration politique des femmes. Parmi ces obstacles, c'est le mode de scrutin, facteur technique important du système électoral que nous privilégions. Ce choix s'explique par l'importance que lui attribue la science politique, mais surtout parce qu'au Québec, les autorités politiques ont notamment utilisé cet argument pour favoriser la réforme du mode de scrutin.

Étude donc de la spécificité de l'exclusion des femmes à partir du mode de scrutin et examen de l'importance relative de ce mécanisme; en d'autres termes il s'agit de voir si le mode de scrutin constitue un obstacle décisif et suffisant du point de vue de l'insertion des femmes.

\section{L'influence des systèmes électoraux}

«Je continue d'affirmer que d'une part la règle du candidat unique par parti et du député unique par comté — la base même

5. Jill Vickers et Janine Brodie, Canadian Women in Politics: An Overview, op. cit. 
du scrutin uninominal - contribue directement à favoriser le candidat sur la candidate et que d'autre part le scrutin d'équipe, sans garantir l'augmentation des candidats élues, la facilite en levant l'hypothèque de l'obligatoire choix entre un homme et une femme» ${ }^{6}$.

L'auteur de ces lignes, André Larocque, secrétaire général associé du Conseil exécutif, a également écrit que «le mode de scrutin actuel interdit mathématiquement une représentation équitable des femmes à l'Assemblée nationale. L'introduction d'un scrutin de liste ne la garantit pas mais la permet ${ }^{7}$.

Pourtant, le problème n'est-il pas mal énoncé puisqu'en principe aucun mode de scrutin, aucun système de partis, n'interdit mathématiquement une représentation équitable des femmes. Se pose alors la question du but poursuivi par ceux qui, comme André Larocque, utilisent l'argument «féministe» en faveur d'une réforme du mode de scrutin. Question difficile certes, mais dont l'examen n'en est pas moins nécessaire.

Les études comparatives ${ }^{8}$ sur la disparité des candidatures féminines selon les systèmes électoraux nous apprennent que le système partisan est important. Que ce système a certains effets sur les candidatures féminines. Ces effets s'exercent notamment à travers les réseaux politiques qui contrôlent les mises en candidature, puisque ceux-ci fondent leur choix sur les possibilités de succès attribuées aux candidats. Au Canada, des recherches récentes ${ }^{9}$ ont démontré que les femmes rencontrent de nombreuses

6. André Larocque, "Il faut plutôt s'en mêler», Le Devoir, 4 août 1982.

7. André Larocque, "C'est le mode de scrutin actuel qui bloque la représentation des femmes à l'Assemblée", "Le Devoir, 7 juillet 1982.

8. Drude Dahlerup, "Women's Increasing Political Representation - Does it Make a Difference?", Communication présentée dans le cadre d'un séminaire de l'Unesco, Bonn, 25 et 26 octobre 1982 et Maurice Duverger, La participation des femmes à la vie politique, op. cit.

9. Jill Vickers et Janine Brodie, Canadian Women in Politics: An Overview, op. cit. 
difficultés lors de ce choix; même une fois candidates, elles reçoivent moins d'appuis de la part du parti pour se faire élire que leurs collègues masculins ${ }^{10}$. Mais on observe une hausse récente et relative des candidatures féminines. Cette hausse s'explique notamment par la multiplication des formations politiques et par leur tendance relative à présenter des candidates en plus grand nombre. Néanmoins, c'est au sein des grands partis que les candidates semblent avoir le plus de chances d'être élues. Certes, ces derniers boudent les candidatures féminines et les femmes y demeurent sous-représentées. Mais ces dernières candidates des grands partis ont de bonnes possibilités d'être désignées par élection ${ }^{11}$. Une fois élues, elles semblent d'ailleurs plus «ministrables» que les hommes. Ces signes sont encourageants bien qu'amoindris par le fait que dans tous les cas et dans tous les pays, il semble que le pourcentage des femmes parmi les candidats soit toujours supérieur au pourcentage des femmes parmi les élus. Ceci peut signifier que les possibilités pour les femmes d'être élues sont toujours moindres que celles des hommes. Même dans un pays comme la Norvège où, au cours des années 1970, certains partis politiques, entre autres le Parti libéral et le Parti socialiste de gauche, ont instauré un système de quotas. Ce système est à l'effet d'assurer qu'au sein de toutes les structures électives de ces partis la représentation de chacun des deux sexes soit au moins égale à $40 \%^{12}$. Or, des études récentes faites par des sociologues danois tendent à démontrer que même avec un contingentement qui fixe à $40 \%$ la proportion des femmes parmi les candidats, la

10. Ibid.

11. La proportion d'élues est de l'ordre de trois ou quatre. À ce sujet, voir l'article de Pierre Drouilly, "Les femmes et les élections", op. cit.

12. Torild Skard, "Stratégies for Power: Methods and Means by which Women can become Participants, in Making Public Policies», Communication présentée dans le cadre d'un séminaire organisé par la Commission canadienne de l'Unesco et qui s'est tenu à Ottawa les 28 et 29 mars 1982. 
proportion des femmes parmi les élus demeure relativement peu importante.

Peu de femmes donc se présentent au suffrage des électeurs et l'organisation partisane semble constituer un élément important de ce problème. On dénombre toujours très peu de candidatures féminines dans les pays à scrutin majoritaire. Toutefois, dans certains pays où la représentation proportionnelle existe, le nombre de femmes dans les parlements est relativement plus élevé que dans les pays où persiste le scrutin majoritaire. Dans les pays européens les plus peuplés où la représentation proportionnelle était en vigueur, les pourcentages étaient les suivants, au début des années 1980:

$\begin{array}{ll}\text { Danemark } & 23,5 \% \\ \text { Finlande } & 26,0 \% \\ \text { Norvège } & 23,9 \% \\ \text { Suède } & 27,5 \% \\ \text { Belgique } & 9,7 \%{ }^{13}\end{array}$

Par contre, en République fédérale d'Allemagne où il y a un système mixte, le pourcentage de femmes élues se rapproche sensiblement de celui du Québec qui est de 6,6\%. Il est en effet de $8 \%$ au Parlement fédéral et de $6 \%$ dans les parlements des Laënder ${ }^{14}$. Mais peut-on conclure de ces chiffres que le scrutin proportionnel favorise l'entrée des femmes dans les assemblées élues tandis que le scrutin majoritaire la décourage? Certes non, puisque c'est souvent dans les pays où le pourcentage des femmes élues est plus important, que l'aquisition de l'égalité des droits politiques premiers remonte au début du siècle. Dans ces pays, l'intégration des femmes à la population dite active n'est guère

13. Drude Dahlerup, «Women's Increasing Political Representation - Does it Make a Difference?, op. cit. Pour la plupart, les autres données concernant les pays européens proviennent des documents de travail utilisés lors des deux conférences de l'Unesco auxquelles j'ai participé, à Bonn et à Ottawa, en 1982 et 1983.

14. Ibid. 
récente, même si les indices de ségrégation selon le sexe, par profession et par branche d'activité, sont assez élevés ${ }^{15}$. Il faut aussi considérer d'autres facteurs liés à l'organisation de la vie politique. Il est vrai qu'en droit, notre système électoral est démocratique et égalitaire. Mais en fait, les mécanismes et les règles qui lui sont propres défavorisent les femmes, lorsqu'ils ne les excluent pas purement et simplement. À titre d'exemple, rappelons notamment la faible intégration des femmes dans les réseaux politiques et l'absence relative de modèle politique féminin. La quasi impossibilité, pour la majorité des femmes, d'accéder à des responsabilités politiques s'exprime à travers la publicité faite autour des femmes qui «réussissent» en politique. On fait d'elles une exception et on montre ainsi le peu d'intérêt accordé au travail à temps partiel, aux horaires variables, à l'aménagement des conditions de travail, à l'extension des systèmes de garde, au partage des tâches domestiques. Mais, n'est-il pas logique de croire que la spécificité de l'exclusion/inclusion des femmes est partiellement déterminée, d'abord à ce niveau? ${ }^{16}$.

Divers obstacles sociaux se combinent aux obstacles institutionnels et il en résulte une exclusion des femmes des systèmes représentatifs et parlementaires. Au nombre des obstacles institutionnels le mode de scrutin semble avoir une importance relative et limitée puisque tout se passe ailleurs, notamment dans les partis politiques et au sein de l'électorat. En effet, un mode de scrutin ne crée pas les forces sociales non plus qu'il ne modifie le

15. Voir les données récentes de l'OCDE.

16. Entre autres, ces recherches:

Elfriede Bode, "Les conditions structurelles qui déterminent la vie des femmes". Communication présentée dans le cadre d'un séminaire de l'Unesco, Bonn, 25 et 26 coctobre 1982 ,

et

Anne-Marie Daune-Richard, «Travail professionnel et travail domestique. Étude exploratoire. sur le travail et ses représentations au sein des lignées féminines", Laboratoire d'economic et de sociologie du travail, Aix-en-Provence, 198. 
comportement de l'électorat. Ce sont plutôt ces forces sociales qui modèlent le système de partis, tandis que le système électoral n'interfere que par la suite. Cette vérité fondamentale a été mise de l'avant lors du débat entre Georges Lavau et Maurice Duverger sur les relations entre systèmes électoraux et partis politiques, au début des années $1950^{17}$. Ceci signifie, pour les groupes et les forces en émergence, la nécessité de franchir et ce, préalablement aux élections, des seuils qualitatifs et quantitatifs. En effet, ce sont d'abord les réaménagements des courants d'opinion qui donnent lieu à des modifications des forces politiques; le mode de scrutin n'exerce qu'ensuite des effets limités. Dans le cas du Québec et en ce qui concerne les femmes, l'argument féministe des autorités politiques à l'appui de la réforme du mode de scrutin semble fallacieux. Puisque dans l'hypothèse de la proportionnelle régionale modérée, une augmentation appréciable des femmes à l'Assemblée nationale ne peut se faire que si les femmes sont regroupées dans une organisation relativement structurée, et pour laquelle une certaine audience au plan régional existe. Mais pour l'instant le mouvement féministe québécois est diffus et, ne s'oriente guère vers cette forme d'institutionnalisation. D'ailleurs, selon des études prospectives, pour qu'un parti féministe réussisse à faire élire quelques membres, il lui faudrait obtenir plus de dix pour cent des suffrages et ce, quel que soit le mode de scrutin retenu parmi ceux proposés par les différents gouvernements depuis 20 ans ${ }^{18}$.

17. Voir les précisions données par Maurice Duverger dans l'ouvrage Institutions politiques et droit constitutionnel, op. cit., 160.

18. Le lecteur intéressé par l'étude des modes de scrutin peut consulter le document intitulé Les modes de scrutin au Québec 1970-1982, préparé par le Service de recherche et de documentation de la Bibliothèque de l'Assemblée nationale du Québec, 1983. Sur les données prospectives voir André Bernard, "Les limites et possibilités des projets de réforme du mode de scrutin présentement à l'étude au Québec", Montréal, colloque sur la réforme du mode de scrutin, mars 1983. 


\section{Système représentatiflsystème politico-administratif}

Les femmes sont partiellement admises dans le système représentatif en général et ceci n'est pas à mettre au compte des systèmes électoraux en particulier. Au Québec et au Canada, les femmes ont accédé à certains lieux et à certains niveaux de la vie parlementaire et représentative, en dépit, nous dit-on, d'un mode de scrutin qui leur est hostile. Les progrès ainsi réalisés sont certains mais demeurent extrêmement limités.

Sans doute les femmes continueront-elles de progresser en augmentant leur représentation numérique. En tout cas c'est ce que suggèrent certains indices ${ }^{19}$. Mais notre propos originel, soit la spécificité de l'exclusion/inclusion des femmes hors et dans le système politique, dépasse en importance celui de leur place au sein des institutions parlementaires et représentatives. Précisément, cette question réactive un débat déjà ancien, celui de la place de l'institution représentative dans le système politique. En effet, l'approche institutionnelle classique fait des institutions représentatives et parlementaires une force dominante, une force audessus des autres. Un pouvoir s'organise qui leur procure des organes et la lutte pour le pouvoir coïncide avec la lutte pour le contrôle des institutions.

Mais plus que jamais, la vie politique moderne ne saurait se résumer à la vie parlementaire et représentative. Le Parlement n'occupe plus la totalité du pouvoir. Il semble bien qu'un glissement soit en train de s'effectuer au profit de relais de types bureaucratiques et administratifs. Un tel processus oblige à questionner une vision trop institutionnelle du champ politique. En effet, le passage du système parlementaire et représentatif à un système bureaucratique et administratif déplace sinon restreint le champ de l'élite et rend plus ardues les luttes pour le pouvoir. Ces luttes se structurent

19. Entre autres, l'augmentation de leur participation dans les syndicats et dans les partis politiques. 
autour de la montée de la puissance administrative, autour de «l'administrativisation» de la vie politique.

Mais alors, ne faut-il pas désormais considérer l'administration comme un acteur politique? Une nouvelle mise en relation du politique et de l'administratif ne s'impose-t-elle pas? Tous ces changements qui restructurent le politique, ne constituent-ils pas de nouveaux obstacles à l'intégration politique des femmes? Du point de vue de l'insertion des femmes quelle est l'importance relative de ces nouveaux obstacles? Ceux-ci ne jouent-ils pas en conjonction avec d'autres, notamment les facteurs institutionnels? On mesure l'importance de ces questions à l'une des difficultés rencontrées pour cerner ce problème: où sont les femmes dans les processus réels de décision? Ces difficultés relèvent notamment de la science du politique. Dans l'ensemble, celle-ci a en effet considéré la question féministe sous l'angle quasi-exclusif du plus ou moins grand renforcement du droit politique premier des femmes. Mais, depuis que l'exercice du pouvoir semble s'être déplacé, sous l'effet de l'élargissement sans cesse croissant du territoire des hauts fonctionnaires, le problème de l'insertion des femmes au sein des institutions démocratiques procède aussi de la mise en lumière de leur exclusion/inclusion hors et dans ce processus décisionnel.

\section{Les femmes dans le processus décisionnel}

Il est désormais reconnu que l'administration impulse et contrôle tous les projets d'envergure et, de ce fait, régule une bonne partie du processus politique. Dans ce nouveau contexte, les administrations publiques constituent un moyen privilégié pour les femmes d'accéder aux niveaux décisionnels. D'ailleurs, des études canadiennes récentes ${ }^{20}$ démontrent que les attributs

20. Janine Brodie et Jill Vickers, Canadian Women in Politics: An Overview, op. cit., William W. Lammers et Joseph L. Nyomarkay, "The Canadian Cabinet in Comparative Perspective», Revue canadienne de science politique, XV:11, mars 1982, 29-46. 
des personnes qui, au sein des cabinets, exercent une grande influence sur le processus décisionnel, sont essentiellement d'ordre administratif. Précisément, la plupart d'entre eux ont commencé leur carrière dans les hautes instances administratives de l'appareil central d'autorité avant d'être intégrés au cercle étroit du cabinet, lieu où s'effectuent les choix politiques.

Au sein de l'administration canadienne le nombre de femmes occupant un poste au sein de la catégorie haute direction a augmenté au cours des années 1970 . Cette période s'est caractérisée par le développement et la spécialisation de l'appareil d'État central, d'où une forte augmentation du nombre de fonctionnaires. En effet et pour ces années, c'est dans le secteur public fédéral que l'augmentation relative a été la plus importante. Environ 60000 nouveaux postes ont alors été créés ${ }^{21}$. Pour sa part, la catégorie haute direction a augmenté de $150 \%{ }^{22}$.

Précisément, 441 personnes ont été nommées à un nouvel emploi au sein de cette catégorie, dont 43 sont des femmes. C'est bien peu pour la catégorie la plus prestigieuse de la fonction publique, catégorie qui procure influence et autorité sur les politiques et les programmes. À la fin de l'année 1979 plus de la moitié des femmes cadres sont classées au niveau le plus bas de la catégorie, tandis que 44 d'entre elles (sur un total de 50 en 1979) se retrouvent aux niveaux un et deux. Leur répartition ministérielle pour l'année 1979 s'établit ainsi: dans onze ministères (sur un total de 26), il n'y a aucune femme dans la catégorie haute direction; dans cinq autres, il y en a une seule. Finalement, on ne compte que dix

\footnotetext{
21. 'D'après les données fournies par la Commission de la fonction publique dans ses Rapports annuels.

22. Ibid.

Si nos données s'arrêtent à 1980 , c'est qu'au cours de cette année, une nouvelle catégorie Gestion a été mise en place, formée de deux sous-groupes. La formation des deux sousgroupes n'étant pas encore terminée, il est donc difficile de mesurer si des changements importants ont eu lieu. Pour de plus amples détails, on peut consulter les Rapports annuels de la CFP.
} 
ministères où la présence des femmes cadres n'est pas purement symbolique. Au sein des structures décisionnelles, elles sont près d'une quinzaine, ainsi réparties: deux au Bureau du conseil privé, dix au Conseil du Trésor, deux aux Finances ${ }^{23}$. Mais leur répartition ministérielle n'est pas uniforme. En effet pour l'année 1979, les femmes cadres sont totalement absentes des ministères à vocation technique et économique. La moitié d'entre elles travaillent au sein des ministères sociaux et culturels. Quant aux autres, elles sont réparties au sein des ministères politiques (Secrétariat d,État, Agence canadienne de développement international notamment). Une telle distribution n'est sans doute pas anodine. Elle l'est d'autant moins qu'une des stratégies actuellement mises de l'avant par le gouvernement fédéral consiste à créer des entreprises publiques à caractère économique. En fait, 69\% des sociétés de la couronne existantes relèvent de ministères dont la vocation dominante est économique ${ }^{24}$. Les personnes qui siègent sur les conseils d'administration de ces structures décentralisées sont nommées par décret du conseil. Une étude du Conseil consultatif canadien de la situation de la femme nous apprend qu'en 1980 et pour 1248 postes nominatifs, 186 ont été attribués à des femmes, soit $15,1 \%$. Là aussi, les femmes semblent être plus nombreuses au sein des organismes qui dépendent de ministères dont la vocation est d'abord sociale ou politique.

Dans les organismes dont l'autorité relève de ministères économiques, le pourcentage des nominations féminines est inférieur à $10 \%$. Malheureusement, les données disponibles ne permettent

23. Le nombre élevé de femmes cadres au sein du Conseil du Trésor s'explique sans doute par le fait que cet organisme a la responsabilité de surveiller la mise en place des programmes ministériels d'égalité des chances. Voir sur cette question:

Carolle Simard, L'administration contre les femmes, Montréal, Boréal Express, 1983.

24. Carolle Simard, "L'État administratif ou les enjeux des transformations administratives récentes: le cas canadien", Montréal, Cabiers de l'ACFAS, no 16, 1983, 315-329.

25. Lise Champagne, "Il y en a, mais si peu», Ottawa, CCCSF, 1980. 
guère d'évaluer si le niveau hiérarchique des postes occupés par les femmes est plus élevé dans certains types d'organismes. Cependant, on ne peut ignorer que plus de la moitié des femmes nommées à plein temps l'ont été dans deux organismes seulement, soit la Commission de la citoyenneté et le Conseil consultatif canadien de la situation de la femme. En outre, moins de $10 \%$ de ces femmes nommées à plein temps occupent des postes de cadre supérieur. Ces treize femmes sont fort inégalement réparties dans l'échelle hiérarchique puisque seulement deux sont classées au niveau le plus élevé, soit celui de sous-ministre. Quant aux femmes nommées à temps partiel, aucune n'a atteint le sommet de l'échelle hiérarchique.

Même si depuis l'an dernier une femme fait désormais partie du comité fédéral des priorités, il n'est pas dit que les femmes soient intégrées pour autant aux différentes étapes du processus de la prise de décision. Toutefois, elles sont de plus en plus nombreuses à travailler dans le secteur public. Mais peut-être là plus qu'ailleurs elles demeurent pour la plupart limitées au monde des petits fonctionnaires, aux emplois plus ou moins interchangeables ${ }^{26}$.

L'institution parlementaire et représentative ne résume plus la vie politique, d'autant que la fonction de député est devenue un emploi relativement peu important. La montée du pouvoir exécutif qui s'appuie sur la puissance administrative semble aller de pair avec la perte de contrôle des parlements. Tant l'initiative des lois que le pouvoir d'en disposer de manière libre échappent à ces derniers. Dès lors que s'instaure un nouveau système de prise de décision, c'est la place des femmes dans ce nouveau système qu'il convient d'appréhender. Sans doute, les indices retenus pour le secteur fédéral ne nous permettent guère de conclure qu'au sein des nouveaux canaux d'influence les femmes sont encore

26. Carolle Simard, L'administration contre les femmes, op. cit. 
moins nombreuses que dans les parlements. Mais, ces indices nous montrent cependant clairement que là aussi, les hommes continuent de monopoliser les meilleures places puisque les positions stratégiques au sein des différents comités demeurent sous le contrôle étroit d'une minorité d'hommes. Certes, les lieux de l'exercice du pouvoir se déplacent mais, n'est-il pas logique de supposer que tous ces changements ne favorisent guère les femmes? Comment croire en effet, qu'au nombre des objectifs poursuivis par ceux qui organisent ces changements, figure la déperdition du processus d'exclusion séculaire des femmes du politique? Cette même interrogation guide l'examen des données concernant les femmes dans la fonction publique du Québec. Bien que limitées, ces données permettent néanmoins d'établir certaines corrélations. Au gouvernement du Québec, les femmes cadres sont peu nombreuses. En 1980, elles représentent moins de $2 \%$ des emplois de cadres supérieurs ${ }^{27}$. Elles sont regroupées dans un nombre limité de ministère puisque dans 36 ministères ou organismes sur un total de 48 , le nombre de femmes cadres varie de zéro à $u^{28}$. Plus de la moitié des femmes cadres travaillent dans une dizaine de ministères et elles sont plus nombreuses qu'ailleurs dans trois ministères: Affaires sociales, Éducation, Justice ${ }^{29}$.

Les données relatives aux femmes à l'emploi du gouvernement mais dont le statut n'est pas régi par la loi $50^{30}$ sont aussi intéressantes, puisqu'elles nous renseignent sur les sous-ministres adjointes ou associées, les secrétaires générales associées, les directrices de cabinet et enfin, les présidentes d'organismes. L'organisation gouvernementale compte peu de sous-ministres adjointes. Elles

27. D'après Présence des femmes et des bommes dans la fonction publique du Québec, vol. 2, Québec, ministère de la Fonction publique, 1980.

28. Ibid.

29. Ibid.

30. Rappelons que la loi 50, adoptée en 1978 , précise les conditions d'embauche et de promotion des fonctionnaires, à l'exception des sous-ministres en titre, des sousministres adjoints et associés, et du personnel des cabinets ministériels. 
sont 8 sur un total de 110 . Si le nombre de sous-ministres adjoints est passé de 74 à 110 depuis 1976 (une augmentation de 48\%), les femmes en ont bénéficié puisqu'elles n'étaient que 3 en 1977. Certes, leur nombre a presque triplé mais leur pourcentage demeure très bas. $\mathrm{Si}$, dans près de $30 \%$ des ministères on compte une sous-ministre adjointe ou une secrétaire générale associée ${ }^{31}$, aucun ministère par contre, n'en a plus d'une. Même dans certains "gros ministères» où il y a cinq sous-ministres adjoints ou plus, on n'a pas encore nommé de femme, notamment aux ministères de l'Agriculture, du Conseil exécutif, de l'Énergie et Ressources, des Finances, de l'Industrie, Commerce et Tourisme, de la Maind'oeuvre et Sécurité du revenu, du Revenu et des Transports. Les directrices de cabinet sont moins nombreuses encore. Il y en a 2 sur un total de 32, à peine $6 \%$. Mais leur nombre a doublé depuis 1977 , année où une seule femme était directrice de cabinet à la Condition féminine.

Enfin, les femmes présidentes d' organismes (Conseils, Régies, Bureau, Sociétés) sont au nombre de 7 , moins de $6 \%$. Toutes, sauf une, dirigent des organismes dont la mission dominante est tantôt sociale, tantôt culturelle. En effet, les femmes président ces organismes: Société du Grand Théâtre de Québec, Conseil du statut de la femme, Conseil des affaires sociales et de la famille, Office des services de garde à l'enfance du Québec, Commission des droits de la personne, Régie de l'assurance-automobile.

Il est certain que ces données chiffrées ne résument pas tout le problème de la spécificité de l'exclusion/inclusion des femmes hors et dans le processus décisionnel. Les lieux de l'exercice du pouvoir se déplacent, changent. Pourtant, les réformes proposées par les autorités politiques et qui sont censées intégrer les femmes à la vie politique ne peuvent cacher les liens plus profonds, plus

31. D'après la «Liste de direction des ministères", ministère du Conseil exécutif, Cabinet du Premier Ministre, décembre 1982. 
déterminants où sont fixées les règles du jeu. Le processus de décision politique dépend, en dernier ressort, de la structuration du pouvoir. Ce n'est pas un hasard si les premiers politologues sont des philosophes qui ont essayé de comprendrre les mécanismes de dévolution du pouvoir. On sait aujourd'hui que leurs théories ne sont pas toujours vérifiées dans les faits. Pourtant leur questionnement demeure essentiel. D'où vient le pouvoir? Pour qui est le pouvoir? Par la suite, les sociologues ont succédé aux philosophes. Ils ont essayé de situer le phénomène du pouvoir dans son rapport aux classes sociales. Encore aujourd'hui, le grand débat de la science politique reste de savoir si le pouvoir peut être qualifié ou non de bourgeois. Mais à certains égards, le débat engagé est dans une impasse. La raison en est peut-être que ce nouveau système d'explication du politique se situe à un niveau trop général. Et si d'autres données devaient être prises en compte pour saisir la spécificité du pouvoir moderne? Parmi l'une d'elles se trouve être celle de la différence sexuelle. La politique de la bourgeoisie n'a-t-elle pas été d'abord celle des bourgeois? Certes, le discours et certaines luttes des révolutionnaires socialistes ont su donner une place plus conséquente aux femmes. Mais le socialisme n'a pas non plus libéré les femmes. En fin de compte notre histoire est au masculin, notre politique aussi. N'est-il pas temps que la science du politique échappe aux généralisations, au totalitarisme sexuel? Le pouvoir n'est-il pas sexué?

\section{Système politico-administratiflscience du politique}

La question des obstacles et des limites à l'insertion des femmes dans le système politique remet en cause le politique, conteste la connaissance du politique. Cette question déstabilise la science du politique puisqu'elle implique une restructuration du politique. 
Car, dans les sociétés où le pouvoir politique est institutionnalisé, où il est exercé à travers une administration spécialisée et hiérarchisée ${ }^{32}$ ne s'est jamais posé, du moins jusqu'à tout récemment, la question du sexe du pouvoir. Certes, les femmes ne sont pas les seules exclues mais l'originalité des politologues féministes est peut-être de montrer qu'en science politique, il n'y a pas de sujet politique différencié (exception faite de quelques études de sociologie électorale). Il n'existe que des sujets politiques "neutres», c'est-à-dire non marqués sexuellement. Mais l'étude des réalités, notions et valeurs impliquées par la chose politique, entre autres «les rapports politiques, le pouvoir politique, les institutions politiques, les partis politiques, les événements politiques, les idées politiques, les forces politiques, la vie politique, les révolutions politiques» ${ }^{33}$ entraîne une restriction énorme du champ de connaissance du politique. Ce champ se limite aux rapports États/ gouvernants/gouvernés. Certes, la sociologie a déjà posé et analysé comme un fait social la différence des sexes. Mais ce fait est aussi d'ordre politique puisqu'il induit des rapports de domination, des rapports de pouvoir. Et soutenir qu'entre les sexes coexistent des rapports de pouvoir, c'est suggérer qu'il y a des dominants et des dominés; c'est aussi remettre en cause la neutralité du champ politique, son universalité. Mais c'est surtout énoncer le problème en termes opposés: le champ politique et l'espace politique ont été définis et construits à partir d'un mode de valeurs et de pensées, d'un système de normes et de règles, lesquelles nient la différence politique des sexes; ne sont définis comme politiques que les problèmes spécifiquement liés à ce champ et à cet espace. Ainsi se profile derrière l'unisexe politique, le pouvoir mâle, la

32. Jean-William Lapierre, dans son ouvrage intitulé Vivre sans État? Paris, Seuil, 1977, distingue neuf degrés de différenciation du pouvoir politique. Celui qui s'exerce à travers une administration spécialisée et hiérarchique constitue, selon la typologie de l'auteur, la 9e degré.

33. Dans La science politique, Paris, PUF, Que sais-je?, no 909, 1969, Marcel Prélot définit en ces termes la science politique. 
norme universelle. Le seul modèle admissible étant celui que les hommes ont défini et érigé en système, lui seul et pas un autre. En politique plus quailleurs, la réalité s'exprime à travers un univers essentiellement masculin. Ce qui est associé dans la réalité reflète les idées que s'en font les uns et les autres et les faits se plient à cette vision de l'ordre politique. Les femmes s'autoexcluent d'elles-mêmes tandis que les hommes ont le sentiment, né de la conviction, que l'univers politique leur appartient, à eux seuls. Quant aux femmes qui se fourvoient en politique, celles qui réussissent «bénéficient d'un curieux privilège: elles changent de sexe et se voient attribuer les qualités viriles grâce auxquelles il est possible de dissimuler leur travers de n'être que des femmes. Et ceci au cri de "quel homme cette femme!» ${ }^{34}$.

Depuis que le pouvoir politique est institutionnalisé, depuis que l'ordre politique se construit à travers le désordre des luttes successorales, le système politique demeure réservé aux hommes en raison d'un double prétexte: selon certains, il correspond au tempérament masculin et il est contraire à la «nature féminine». Car, consciemment ou non, les hommes y ont imposé les valeurs dont ils ont le monopole, qui les caractérisent, et auxquelles s'ajustent les exigences du pouvoir et de l'ordre. Ce ne sont donc pas les femmes qui refusent cet ordre; c'est tantôt cet ordre qui nie le désordre des valeurs pour lesquelles les femmes sont censées être réputées - fait de culture et non de nature; c'est tantôt cet ordre qui nie les ordres contraires que les femmes menacent d'introduire.

Mais de quelles normes opposées s'agit-il et quel est l'effet de leur coexistence sur le système politique? Il n'est guère facile de répondre à ces questions sans une certaine reconstruction, c'està-dire sans une remise en cause de l'objet d'étude. Ceci n'est

34. Jacques Baguenard, Jean Maisondieu et Léon Métayer. Les hommes politiques n'ont pas d'enfant, Paris, PUF, 1983, 114. 
possible qu'en transposant au plan théorique le refus du pouvoir mâle en tant que norme et par la réédification d'une autre réalité, celle des faits. Une telle reconstruction ne peut se faire, du moins dans un premier temps, qu'à travers un processus inductif et dont l'objet est la découverte des faits.

La discipline-mère qu'est la science politique n'a guère de place pour les femmes, ni guère de place pour les questions qu'elles posent. Ces questions procèdent essentiellement des conditions de vie qui les caractérisent, conditions de vie marquées par de profonds bouleversements, aux plans juridique et financier notamment. Car, depuis que les femmes ont été conviées à partager le sort des hommes salariés les plus démunis, elles ont hérité de charges supplémentaires, mais sans contrepartie réelle. Poser le problème du point de vue des questions induites par les femmes implique le refus d'une prise en compte des schémas scientifiques pré-établis et à travers lesquels a été intégrée ou pas, la variable femme. Cela suppose une interrogation autre, laquelle procède de ce qu'il est convenu d'appeler le «vécu» des femmes. À titre d'exemple, si l'on s'intéresse au problème de la participation des femmes à la vie politique, on ne peut éviter de considérer qu'il s'agit bien là d'une troisième journée de travail. À l'opposé des hommes, les activités des femmes sont souvent traversées de ruptures et marquées de contradictions. Constamment et partout illégitimes, les femmes s'ajustent sans cesse à des normes qu'elles ne contrôlent pas. Elles sont tolérées tant et aussi longtemps qu'elles ne mènent pas leur propre politique; tant et aussi longtemps qu'elles souscrivent à la stratégie de l'un ou l'autre clan. Question cruciale qui ébranle l'opposition droite-gauche, opposition qui ne saurait prendre valeur d'indices plus longtemps, l'anti-féminisme de la droite renvoyant au prétendu féminisme de la gauche. De part et d'autre, la profession de foi est sans cesse renouvelée et les idées ainsi exprimées tiennent lieu de politique. Sans cesse grippée, cette politique a notamment pris la forme de reconnaissances juridiques et bureaucratiques. 
Cette intégration normative des faits de liberté s'effectue par le biais de programmes de tous ordres. Le système politico-administratif gère les institutions et ces dernières servent de litière aux discriminations. Pour sa part et plutôt bien que mal, la société civile les digère.

Sans doute, l'origine du pouvoir politique demeure trouble, cependant que les structures à travers lesquelles ils s'exercent sont relativement bien circonscrites. Tout au moins ceci est-il juste pour les données institutionnelles. Quant aux données stratégiques, elles nous instruisent sur les luttes plutôt âpres auxquelles se livrent les hommes et quelques femmes, tantôt pour garder le pouvoir, tantôt pour le conquérir. En ce sens, l'étude des femmes et de la politique, des femmes et du pouvoir, des femmes et des institutions, pose à la science un problème énorme. Précisément, une telle étude conduit à questionner le paradigme dominant dans son rapport au réel, puisque ce système d'explication renvoie obligatoirement à une problématique de l'absence, de la marginalité, de la passivité et du conservatisme. Les données existentielles demeurent irrecevables. La classification traditionnelle en vigueur au sein du système politique ne peut les prendre en compte. Après avoir fait une partition entre sphère publique et sphère privée et convenu que le pouvoir domestique ne peut être politique, il fallait désormais s'assurer, à travers les institutions et les règles, du pouvoir d'un sexe sur l'autre. N'a-t-on pas tout organisé pour que les faits se plient à ces exigences et notamment la science du politique? Car si l'autorité de l'État s'exprime à travers la force qu'elle exerce, elle se traduit également dans les idées qu'elle exprime. La science du politique façonne la légitimité des hommes politiques; elle cautionne l'État dans son universalité et sa neutralité

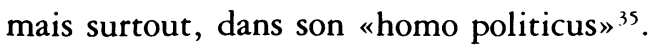

35. Ibid. 
Au-delà de l'importance respective et relative des obstacles et des limites à l'insertion des femmes dans le système politique, il est logique de croire que si les femmes continuent de refuser le politique ${ }^{36}$, c'est sans doute pour d'autres raisons. Structurellement exclues du politique les femmes témoignent, par leurs luttes, du peu d'intérêt qu'elles ont à jouer le jeu de leur exclusion/ inclusion conjoncturelle. Au-delà de son caractère radical, cette prise de position des femmes enseigne que non seulement le pouvoir est structurellement masculin mais que tous les changements qui s'opèrent aujourd'hui ne tendent qu'à renforcer cette caractéristique. Aussi, si les femmes veulent des changements, il leur reste sans doute à en inventer les formes, les lieux et les stratégies.

36. Yolande Cohen, «Réflexion désordonnant les femmes du pouvoir» dans Femmes et politiques sous la direction de Yolande Cohen, Montréal, éd. du Jour, 1981, 193-227. 